\title{
The AGN Content of Hard X-ray Surveys
}

\author{
Andrea Comastri \\ Osservatorio Astronomico di Bologna, via Ranzani 1, I-40127 Bologna, \\ Italy \\ Cristian Vignali \\ Dept. of Astronomy and Astrophysics, Penn State University, 525 \\ Davey Lab - University Park, PA 16802, USA \\ Marcella Brusa \\ Dipartimento di Astronomia, Universita' di Bologna, via Ranzani 1, \\ I-40127 Bologna, Italy \\ on behalf of the HELLAS and HELLAS2XMM consortia ${ }^{1}$
}

\begin{abstract}
.
Multiwavelength observations of the hard X-ray selected sources discovered by BeppoSAX, Chandra and XMM-Newton surveys have significantly improved our knowledge of the AGN population. The increasing number of $\mathrm{X}$-ray obscured AGN so far discovered confirms the prediction of those AGN synthesis models for the X-ray background based on the Unified scheme. However, follow-up optical observations of hard Xray selected sources indicate that their optical properties are quite varied and the simple relations between optical and X-ray absorption are by no means without exception. Moreover there is evidence of a substantial number of luminous X-ray sources hosted by apparently normal galaxies. In this paper the results obtained from multiwavelength observations of hard X-ray selected sources discovered by BeppoSAX and XMM-Newton are presented and briefly discussed.
\end{abstract}

\section{Introduction}

A large fraction of the energy density contained in the cosmic $\mathrm{X}$-ray background spectrum (XRB) is accounted for by the summed contribution of Active Galactic Nuclei (AGN) if most of their high-energy radiation integrated over cosmic time is obscured by gas and dust. Several independent models based on the AGN unification scheme (Setti \& Woltjer 1989, Madau et al. 1994, Comastri et al.

\footnotetext{
${ }^{1}$ HELLAS: F. Fiore, P. Giommi, G. Matt, F. La Franca, G.C. Perola, S. Molendi, R. Maiolino, A. Antonelli; HELlAS2XMM: F. Fiore, A. Baldi, S. Molendi, M. Mignoli, P. Ciliegi, F. La Franca, G. Matt, G.C. Perola, P. Severgnini, R. Maiolino
} 
1995, Gilli et al. 2001) and energetic arguments (Fabian \& Iwasawa 1999) lead to the conclusion that a fraction as high as $80-90 \%$ of the luminosity produced by accretion-powered sources is obscured at almost all wavelengths, emerging only in the hard $\mathrm{X}$-ray band above a few $\mathrm{keV}$. Hard $\mathrm{X}$-ray surveys represent thus the most efficient method to search for and to trace the cosmological evolution of accretion-powered sources. A still unknown fraction of obscured radiation is reprocessed and re-emitted in the far-infrared band. Multiwavelength follow-up observations of hard X-ray selected sources would allow probing where the bulk of obscured accretion power is re-emitted and estimating the AGN contribution to the extragalactic background light in the infrared band.

Thanks to their revolutionary capabilities (arcsec imaging and high-energy throughput) Chandra and XMM-Newton have opened up a new era in the study of the hard X-ray sky. Deep Chandra surveys (Brandt et al. 2001, Rosati et al. 2001) have reached extremely faint fluxes in the $0.5-2 \mathrm{keV}$ and $2-7 \mathrm{keV}$ bands virtually resolving the entire XRB flux at these energies; while relatively deep XMM-Newton exposures (Hasinger et al. 2001, Baldi et al. 2001) have extended by a factor of 50 the sensitivity in the 2-10 and 5-10 keV bands with respect to previous $A S C A$ and BeppoSAX observations. The $\mathrm{X}$-ray source counts and their average spectral properties, which are now probed over several decades of fluxes and energy ranges, appear to be consistent with AGN synthesis model predictions. Although remarkable results have been obtained so far, deep Chandra and XMM-Newton surveys are limited by small area coverage (less than a quarter of a square degree) and by the extremely faint magnitudes of the optical counterparts, which make the identification of $\mathrm{X}$-ray sources very difficult, if not impossible, even at $8 \mathrm{~m}$ class telescopes (Giacconi et al. 2001, Tozzi et al. 2001, Norman et al. 2001). In order to fully characterize the nature and evolution of the $\mathrm{X}$-ray source population it is customary to complement deep surveys with shallower observations carried out in larger areas (see for example the Einstein Medium Sensitivity Survey: EMSS; Gioia et al. 1990). This approach allows us to minimize the effects of field-to-field fluctuations (the cosmic variance) and makes the optical identification follow-up observations much easier, given the average brighter magnitude of the counterparts.

In this review the results obtained by two large area surveys carried out with BeppoSAX (HELLAS) and XMM-Newton (HELLAS2XMM) are summarized. The main scientific drive of this project is to probe the obscured accretion history of the $\mathrm{X}$-ray Universe. The adopted strategy is a trade off between the hardest $\mathrm{X}$-ray band and the largest area which can be covered with BeppoSAX and XMM-Newton.

\section{The HELLAS View of the Hard X-ray Source Population}

The High Energy Large Area Survey (HELLAS; Fiore et al. 1999, 2001a) has provided, for the first time before the advent of the new $\mathrm{X}$-ray observatories Chan$d r a$ and XMM-Newton, a well-defined and large-area sample of hard (5-10 keV) $\mathrm{X}$-ray selected sources obtained with an imaging instrument (the MECS instrument onboard BeppoSAX). At the flux limit of about $5 \times 10^{-14} \mathrm{erg} \mathrm{cm}^{-2} \mathrm{~s}^{-1}$, the integrated flux of the HELLAS sources accounts for some $20-30 \%$ of the hard 5-10 keV XRB flux (Comastri et al. 2001). 
The optical identification follow-up has been carried out on a subsample of 118 HELLAS sources covering $\sim 55 \mathrm{deg}^{2}$. The details of sample selection and optical identification breakdown are reported in Fiore et al. (2001a, 2001b) and La Franca et al. (2002). About $60 \%$ of the objects have been optically identified either by cross-correlation with public catalogs ( 25 objects) or at $4 \mathrm{~m}$ class telescopes (49 objects). For 13 sources there are no clear counterparts down to $\mathrm{R} \simeq 21$. Even if the optical identifications are dominated by type $1 \mathrm{AGN}$, the relative fraction of type 2 objects (including Seyfert 1.8, 1.9 and emission-line galaxies) vs. type 1 is about 0.40 , considerably higher than the value of 0.25 found in the ROSAT Ultradeep Survey (Lehmann et al. 2001a) and that of 0.20 in the ASCA Large Area Survey (LSS; Akiyama et al. 2000). If one assumes that the 13 "empty" fields host Type 2 AGNs, then the Type $2 /$ Type 1 ratio becomes about 0.7 . This shows the efficiency of revealing obscured $\mathrm{X}$-ray sources in the 5-10 keV band with BeppoSAX.

Although for the majority of the HELLAS sources a "standard" spectral analysis is not possible due to the low photon statistics, the analysis of X-ray colors (hardness ratios) allows us to unambiguously reveal a substantial number of objects with flat and/or absorbed X-ray spectra. The most intriguing result is the presence of hard, presumably absorbed X-ray spectra in objects optically classified as type 1 AGN with a blue optical/UV continuum and broad lines. The number of $\mathrm{X}$-ray absorbed type 1 quasars appears to increase with redshift and/or luminosity (Fig. 1).

In fact, while at redshifts lower than 0.3-0.4 X-ray obscured HELLAS sources are mainly associated with Type 2 AGNs and narrow emission-line galaxies, at higher redshifts the presence of X-ray absorption in a sizeable fraction of Type 1 AGNs represents a new, intriguing issue to be subjected to further Chandra and XMM-Newton observations, clearly suggesting that AGNs properties cannot be exhaustively interpreted without a multiwavelength approach. The decoupling between the optical and X-ray absorption properties of $z>0.3$ 0.4 broad-line AGNs can be explained assuming a dust-to-gas ratio significantly lower than the Galactic value. From a physical perspective, this could imply the presence of a population of dust grains with different properties (e.g., large size) than previously known or thought (Maiolino et al. 2001). Strong X-ray absorption $\left(N_{\mathrm{H}}>10^{23} \mathrm{~cm}^{-2}\right)$ in high-redshift broad-line AGNs has also been suggested to explain the X-ray properties of four objects detected in the course of the ASCA LSS (Akiyama et al. 2000), and recent results from Chandra observations seem to confirm this result at lower X-ray fluxes (Fiore et al. 2000, Akiyama et al. 2001). The discovery of X-ray absorption in optically "normal" broad-line AGNs has important consequences for the AGN synthesis models for the XRB. According to these models, the sources responsible for the XRB must be characterized by a spectral energy density spanning a wide range of luminosities and absorption column densities, in order to reproduce both the XRB spectrum and the source counts in different energy ranges. In particular, the energetically dominant contribution comes from sources around the knee of the $\mathrm{X}$-ray luminosity function $\left(L_{\mathrm{X}} \sim \mathrm{a}\right.$ few $10^{44} \mathrm{erg} \mathrm{s}^{-1}$ at $\left.\mathrm{z}=1\right)$ and with absorbing column densities of the order of $10^{23} \mathrm{~cm}^{-2}$ (Comastri 2000). If one relies on the AGN unified model, these objects, the so-called "QSO2", are expected to be the luminous counterparts of the local Seyfert 2 galaxies with narrow optical emission lines. Despite extensive searches only a handful of candidates have 


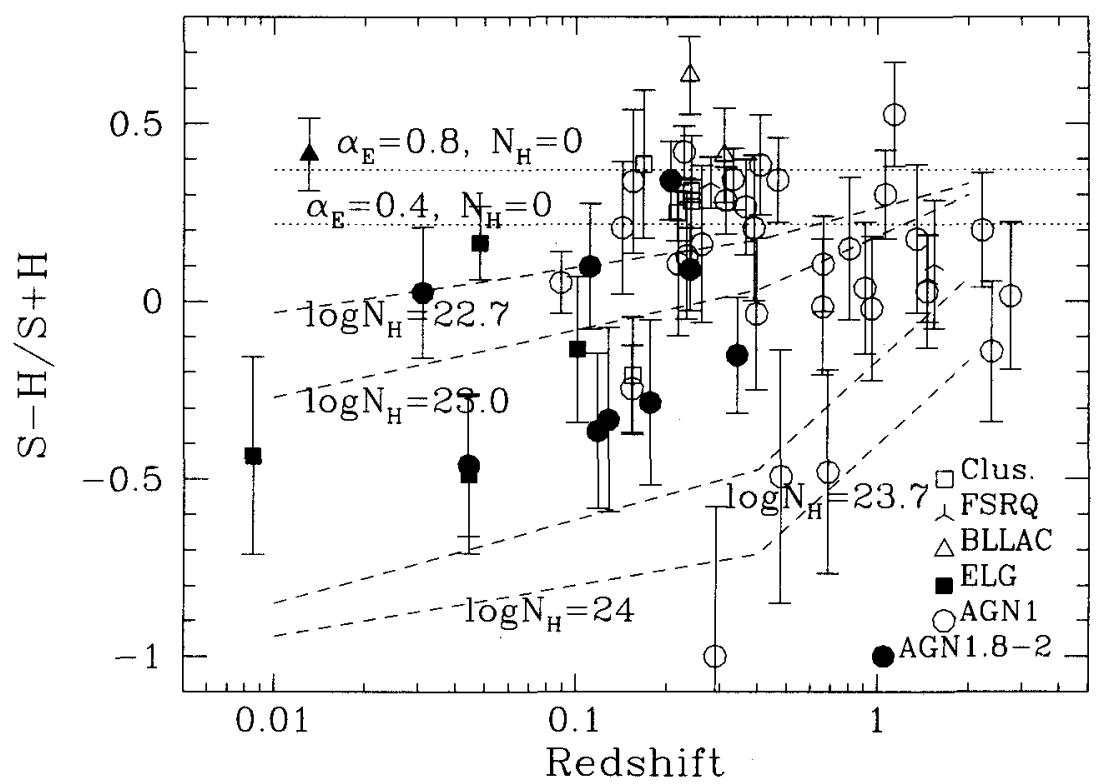

Figure 1. The softness ratio $(\mathrm{S}-\mathrm{H} / \mathrm{S}+\mathrm{H})$ as a function of redshift for the identified sources, where $\mathrm{S}$ and $\mathrm{H}$ are the counts in the soft 1.3-4.5 and hard $4.5-10 \mathrm{keV}$ bands, respectively. The dotted lines show the expected hardness ratio for power law models, while dashed lines the expectations for absorbed power law models with $\alpha=0.8$ and different values of column density at the source redshift.

been found, the most remarkable example being the $z=3.7$ quasar discovered in the Chandra deep field south (Norman et al. 2001). The results obtained by HELLAS suggest that if the statement: obscured Type $2 \equiv$ narrow optical lines is not always true, the moderate/high-redshift absorbed Type 1 objects could have the same role of the so far elusive class of QSO2 in contributing to the XRB (Comastri et al. 2001).

Absorption column densities in excess of the Galactic one, often larger than $10^{22}-10^{23} \mathrm{~cm}^{-2}$, are also derived combining archival ROSAT data with the BeppoSAX ones (Vignali et al. 2001). Interesting enough, this broad-band $\mathrm{X}$-ray analysis has revealed that a fraction of sources which are thought to be absorbed in the hard X-rays are characterized by hard colors also in soft X-rays. This means that, although with lower efficiency, it is possible to pick up absorbed objects in the soft $\mathrm{X}$-rays. It must be noted that some evidence of absorbed objects has also been obtained from the ROSAT Deep and Ultradeep Surveys in the Lockman Hole (Hasinger et al. 1998; Lehmann et al. 2000, 2001a). ROSAT observations of the HELLAS sources suggest that their average spectral properties are not well accounted for by a simple absorbed power-law model. More complex spectra are required in a high number of cases, and this is particularly 
true for very absorbed objects. Additional soft components possibly due to scattered nuclear flux and/or starburst components are required, in most cases involving about $10-50 \%$ of the primary radiation (Vignali et al. 2001). Similar results have been obtained by $A S C A$ (Della Ceca et al. 1999).

Remarkable results have been obtained also by the photometric follow-up observations of a subsample of HELLAS sources. Interestingly enough, the optical and the near-infrared properties of a fraction of intermediate (1.8-1.9) and Type 2 objects (Maiolino et al. 2000), as well as red quasars (Vignali et al. 2000 ), are dominated by the stellar component of the galaxies hosting the (obscured) X-ray active nucleus. This result has straightforward consequences, since it implies that a fraction of sources responsible for the hard XRB may be hosted by normal, passively-evolving galaxies, possibly being missed by previous optical surveys based on color-selection criteria.

\section{From BeppoSAX to XMM-Newton: The HELLAS2XMM Survey}

The HELLAS2XMM survey aims to cover a portion of the redshift-luminosity plane which cannot be probed by deep pencil-beam surveys. The main purpose of this complementary approach is to study the $\mathrm{X}$-ray source populations at fluxes where a large fraction of the hard X-ray cosmic background (HXRB) is resolved $\left(\approx 50 \%\right.$ at $F_{2-10}>10^{-14} \mathrm{erg} \mathrm{cm}^{-2} \mathrm{~s}^{-1}$, see e.g. Comastri 2000 ), but where a) the area covered is as large as possible, to be able to find sizeable samples of "rare" objects; b) the X-ray flux is high enough to provide at least rough X-ray spectral information; and c) the magnitude of the optical counterparts is bright enough to allow, at least in the majority of the cases, relatively high-quality optical spectroscopy, useful to investigate the physics of the sources. Our goal is to evaluate for the first time the luminosity function of hard X-ray selected sources in wide luminosity and redshift ranges. By integrating this luminosity function we will compute the hard X-ray luminosity density per unit volume due to accretion as a function of the redshift. A comparison with the history of the UV luminosity density (proportional to the history of the star-formation) may give us a clue about the correlations between formation and evolution of AGN and supermassive black holes and formation and evolution of galaxies.

At present, the HELLAS2XMM sample, performed using 15 XMM-Newton public observations, consist of 1022,495 , and 100 sources detected down to minimum fluxes of about $6 \times 10^{-16}, 3 \times 10^{-15}, 6 \times 10^{-15} \mathrm{erg} \mathrm{cm}^{-2} \mathrm{~s}^{-1}$ in the $0.5-2,2-10$ and $4.5-10 \mathrm{keV}$ bands, respectively, over an area of about $3 \mathrm{deg}^{2}$ (Baldi et al. 2001). The source counts in these bands are in good agreement with previous determination by other satellites and XMM-Newton itself. In the hard 2-10 and $5-10 \mathrm{keV}$ bands our survey samples a flux range neither accessible by shallower $A S C A$ (Ueda et al. 1999, Della Ceca et al. 1999) and BeppoSAX surveys (Fiore et al. 2001a), which were limited to relatively bright fluxes, nor by deep Chandra (Brandt et al. 2001, Rosati et al. 2001) and XMM-Newton (Hasinger et al. 2001) surveys which are limited by the small area.

Four of the HELLAS2XMM fields were selected for follow-up observations in the optical band using the ESO $3.6 \mathrm{~m}$ and the TNG $3.5 \mathrm{~m}$ telescopes. The selected subsample contains 115 sources with 2-10 keV fluxes between $\sim 8 \times 10^{-15}$ and $10^{-13} \mathrm{erg} \mathrm{cm}^{-2} \mathrm{~s}^{-1}$. Medium-deep $\mathrm{R}$ band images are available for all the 
sources in the sample. Optical counterparts brighter than $\mathrm{R} \simeq 24$ within 5 arcsec from the $\mathrm{X}$-ray position (actually within 3 arcsec in most of the cases; see Fiore et al. $2001 \mathrm{~b}$ ) are present for about $80 \%$ of the sample. At the time of writing, optical spectra have been obtained for 46 out of the 115 sources in our sample. The number of faint hard $\mathrm{X}$-ray selected sources with optical identification is comparable to that obtained in deep Chandra observations (Barger et al. 2001). The HELLAS2XMM sources populate a region of the luminosity-redshift diagram which is barely covered by deep surveys (Fig. 2). A uniform sampling over a large region of the $L_{X}-z$ parameter space is a key issue to compute the luminosity density and evolution of the $\mathrm{X}$-ray sources.

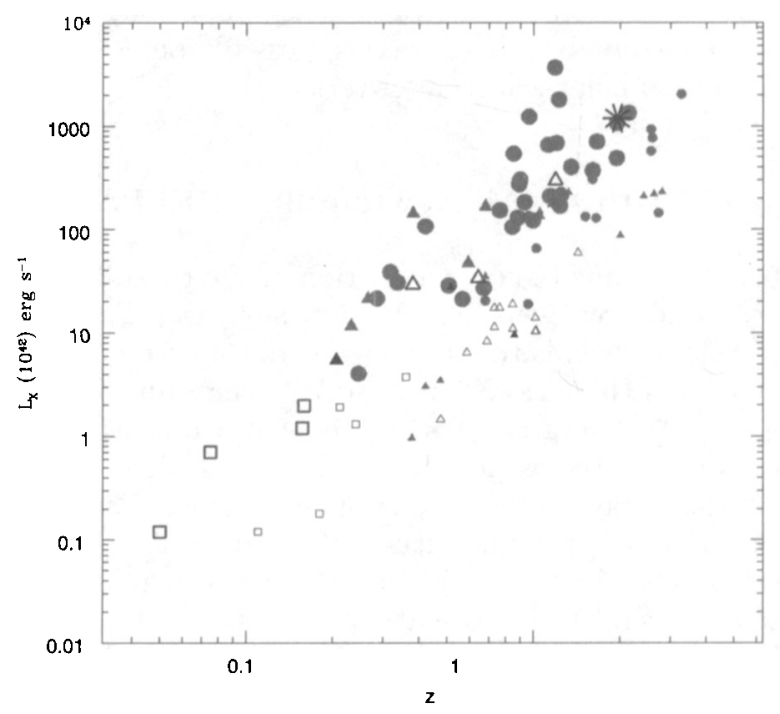

Figure 2. The luminosity-redshift diagram for the HELLAS2XMM sources (big symbols) and the Chandra SSA13, HDFN and A370 deep surveys (smaller symbols, data from Barger et al. 2001). Different symbols identify different source classes: filled circles = broad-line quasars and Sy1; filled triangles = narrow-line AGN; open squares = optically 'normal' galaxies; open triangles = emission-line galaxies; big star $=$ the candidate type 2 QSO at $z=1.955$.

\subsection{Optical Identification Breakdown}

The most interesting results emerging from the optical identification program are the discovery of one $\mathrm{X}$-ray luminous $\left(L_{X} \simeq 10^{45} \mathrm{erg} \mathrm{s}^{-1}\right)$ quasar at $z=$ 1.955 with narrow optical emission lines (FWHM $\lesssim 1000 \mathrm{~km} \mathrm{~s}^{-1}$ ) and of some $\mathrm{X}$-ray luminous but apparently normal galaxies at low redshift. Although type 2 quasars were predicted by AGN synthesis models for the XRB, their space density and optical appearance is still a matter of debate (see the previous paragraph). The present finding suggests that hard X-ray selection coupled with large area provides an efficient method to uncover new objects of this class. More surprising is the presence of X-ray bright sources in the nuclei of otherwise 
passive "normal" galaxies. Their $2-10 \mathrm{keV}$ luminosities, in the range $10^{42-43}$ $\mathrm{erg} \mathrm{s}^{-1}$, are more than one order of magnitude higher than that predicted on the basis of the $L_{X}-\mathrm{L}_{\text {opt }}$ relation of normal galaxies (Fabbiano et al. 1992) and more typical of Seyfert galaxies. Their count ratios indicate a hard $\mathrm{X}$-ray spectrum. A detailed broad-band study has been recently performed on one of these objects (Comastri et al. 2002), suggesting that a heavily obscured, Compton-thick $\left(N_{H}>10^{24} \mathrm{~cm}^{-2}\right)$ AGN may be responsible for the observed properties (Fig. 3).

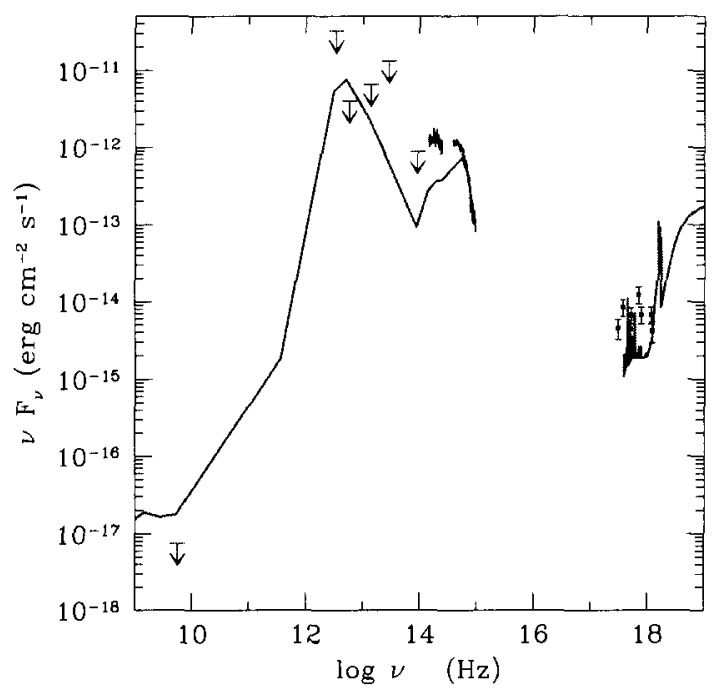

Figure 3. The spectral energy distribution (SED) of the X-ray bright optically quiet galaxy Fiore P3 (see Comastri et al. 2002 for details) is compared with that of the highly obscured Seyfert 2 galaxy NGC 6240 (solid line). The latter SED was normalized to the observed optical spectrum.

\section{2. $\mathrm{X}$-ray to Optical Properties}

The results of photometric observations indicate that $\sim 23 \%$ of the X-ray sources have counterparts with $22<R<24$ and $\sim 17 \%$ with $\mathrm{R}>24$. The identification of the optically faintest counterparts will not probably be feasible even with $8 \mathrm{~m}$ class telescopes, requiring deep multifilter observation to get reliable photometric redshifts. An alternative solution would be to search for redshifted iron $\mathrm{K} \alpha$ lines. Although such an approach may be feasible for the brightest $\mathrm{X}$-ray sources, for the large majority of the objects in our sample the X-ray spectrum cannot be measured due to the low counting statistics. Useful constraints on the nature of the faint $\mathrm{X}$-ray source population might be obtained from the analysis of the already available optical and X-ray fluxes and from an estimate of their average $\mathrm{X}$-ray spectral properties inferred from the hardness ratio analysis. The $\mathrm{R}$ band magnitudes plotted versus the $2-10 \mathrm{keV}$ flux are reported in Fig. 4. 


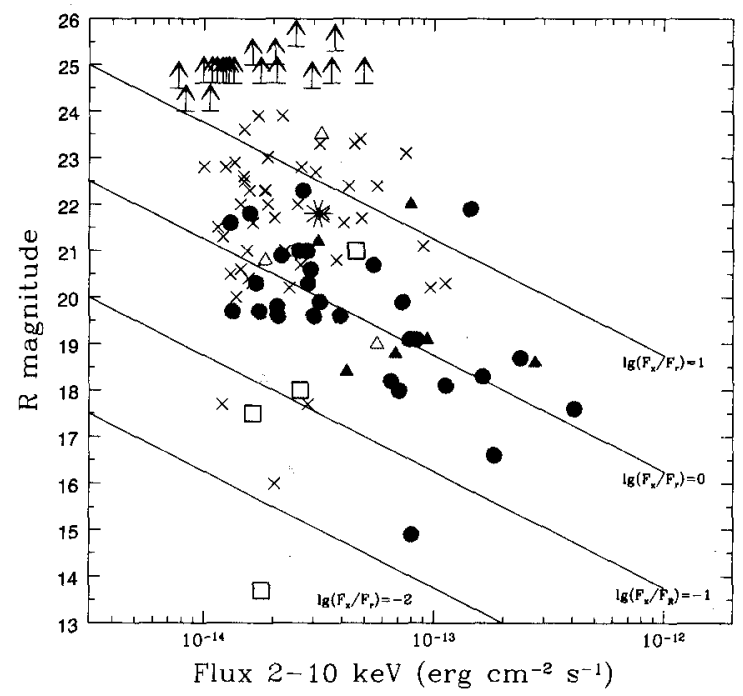

Figure 4. The 2-10 keV X-ray flux plotted against the $\mathrm{R}$ magnitude. The symbols are the same as in Fig. 2, while the crosses represent unidentified sources. Two Galactic stars are also plotted as asterisks. The loci of constant $F_{X} / F_{\text {opt }}$ are reported with the values as labeled.

The AGN identified so far show a relatively well-defined correlation with optical magnitude around $F_{X} / F_{\text {opt }} \simeq 1$. This correlation is similar to that found by $R O S A T$ for soft X-ray selected quasars (Hasinger et al. 1998) and confirmed by Chandra and XMM-Newton observations (Lehmann et al. 2001b) also for hard X-ray selected sources. On the other hand, the X-ray to optical flux ratio of unidentified sources is characterized by a larger scatter and skewed towards higher $F_{X} / F_{\text {opt }}$ values. At faint fluxes there are several objects with $F_{X} / F_{\text {opt }}>$ 10 suggesting the presence of highly obscured AGN. In order to quantify this possibility, we have computed the average hardness ratio as a function of the $\mathrm{X}$-ray to optical flux ratio and the $\mathrm{X}$-ray flux. The entire sample has been divided into bright and faint sources according to the median flux of the survey in the hard X-ray band: $2.5 \times 10^{-14} \mathrm{erg} \mathrm{cm}^{-2} \mathrm{~s}^{-1}$.

The results reported in Fig. 5 indicate a hardening of the $\mathrm{X}$-ray spectrum for those sources with the highest values of $F_{X} / F_{\text {opt }}$, this effect being more pronounced in the faint sample.

\section{Conclusions}

The relatively high number of obscured AGN discovered by BeppoSAX and XMM-Newton makes high-energy large area surveys extremely well suited to 


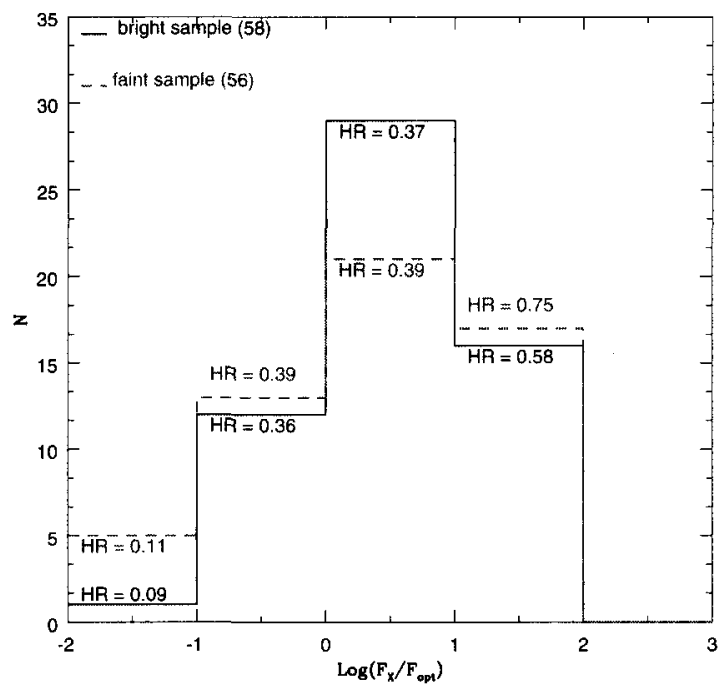

Figure 5. The distribution of X-ray to optical flux ratios for the bright and faint samples. The labeled values correspond to the average hardness ratio in the bin defined as $\mathrm{H}-\mathrm{S} / \mathrm{H}+\mathrm{S}$ where $\mathrm{H}$ and $\mathrm{S}$ are the $2-10 \mathrm{keV}$ and $0.5-2 \mathrm{keV}$ fluxes respectively. Sources with $\log F_{X} / F_{\text {opt }}>$ 1 are grouped in a single bin.

study the physics and the evolution of the sources responsible for the hard Xray background. The hard X-ray sky is populated by AGN with extremely varied broad-band properties. The most important result concerns the optical appearance of X-ray obscured AGN as a function of redshift. In the local Universe a fraction of absorbed objects are associated with apparently normal, early-type galaxies. The lack of any AGN feature in their optical-infrared spectra suggests the presence of buried, probably Compton-thick nuclei. At higher redshift the presence of hard X-ray sources with broad optical lines indicates that the absorbing gas is dust-free. Finally a sizeable fraction of hard X-ray selected sources lacks an optical counterpart at the limit of $4 \mathrm{~m}$ class telescopes. Multiwavelength observations of hard X-ray selected sources allow us to uncover AGN activity in a number of objects which would have not been classified as such on the basis of observations at other wavelengths. Larger samples of hard $\mathrm{X}$-ray selected sources will provide new insights into the physics and the cosmic history of accretion.

Acknowledgments. This research has been partially supported by ASI contracts ARS--99-75 and I/R/107/00, and by the MURST grant Cofin-00-0236. CV also aknowledges the financial support of NASA LTSA grant NAG58107. 


\section{References}

Akiyama, M. et al. 2000, ApJ, 532, 700

Akiyama, M., Ohta, K., Ueda, Y. 2001, ApJ, in press (astro-ph/0111037)

Baldi, A., Molendi, S, Comastri, A., Fiore, F., Matt, G., Vignali, C. 2001, ApJ, in press (astro-ph/0108514)

Barger, A. et al. 2001, AJ, 122, 2177

Brandt, W. N. et al. 2001, AJ, in press (astro-ph/0108404)

Comastri, A., Setti, G., Zamorani, G., Hasinger, G. 1995, A\&A, 296, 1

Comastri, A. 2000, in Proc. Conf. "X-ray Astronomy '999: Stellar Endpoints, AGN, and the Diffuse Background (astro-ph/0003437)

Comastri, A. et al. 2001, MNRAS, 327, 781

Comastri, A. et al. 2002, ApJL, submitted (see also astro-ph/0109117)

Della Ceca, R. et al. 1999, ApJ, 524, 674

Fabbiano G., Kim D. W., Trinchieri G., 1992, ApJS, 80, 531

Fabian, A. C., Iwasawa, K. 1999, MNRAS, 303, L34

Fiore, F. et al. 1999, MNRAS, 306, L55

Fiore, F. et al. 2000, New Astronomy, 5, 143

Fiore, F. et al. 2001a, MNRAS, 327, 771

Fiore, F. et al. 2001b, Proc. of the ESO/ECF/STSCI Workshop on "Deep Fields", Garching October 2000 (astro-ph/0102041)

Giacconi, R. et al. 2001, ApJ, 551, 624

Gilli, R., Salvati, M., Hasinger, G. 2001, A\&A, 366, 407

Gioia, I. M. et al. 1990, ApJS, 72, 567

Hasinger, G. et al. 1998, A\&A, 329, 482

Hasinger, G., Altieri, B., Arnaud, M. et al. 2001, A\&A, 365, L45

La Franca, F. et al. 2002, ApJ, submitted

Lehmann, I. et al. 2000, A\&A, 354, 35

Lehmann, I. et al. 2001a, A\&A, 371, 833

Lehmann, I., Hasinger, G., Murray, S. S., Schmidt, M. 2001b, Proc. of the Symposium "X-rays at Sharp Focus", in press (astro-ph/0109172)

Madau, P., Ghisellini, G., Fabian, A. C. 1994, MNRAS, 270, L17

Maiolino, R. et al. 2000, A\&A, 355, L47

Maiolino, R. et al. 2001, A\&A, 365, 2

Norman, C., 2001, ApJ, submitted (astro-ph/0103198)

Rosati, P. et al. 2001, ApJ, in press (astro-ph/0110452)

Setti, G., Woltjer L. 1989, A\&A, 224, L21

Tozzi, P. et al. 2001, ApJ, in press (astro-ph/0103014)

Ueda, Y. et al. 1999, ApJ, 518, 656

Vignali, C., Mignoli, M., Comastri, A., Maiolino, R., Fiore, F. 2000, MNRAS, 314, L11

Vignali, C., Comastri, A., Fiore, F., La Franca, F. 2001, A\&A, 370, 900 